\title{
Management of hydrocephalus in patients with leptomeningeal metastases: an ethical approach to decision-making
}

\author{
Nayan Lamba ${ }^{1,2} \cdot$ Tim Fick $^{3} \cdot$ Rhishi Nandoe Tewarie ${ }^{4} \cdot$ Marike L. Broekman $^{1,4,5,6}$ (])
}

Received: 10 June 2018 / Accepted: 7 July 2018 / Published online: 18 July 2018

(c) The Author(s) 2018

\begin{abstract}
Purpose Leptomeningeal metastases (LM) are a rare, but often debilitating complication of advanced cancer that can severely impact a patient's quality-of-life. LM can result in hydrocephalus (HC) and lead to a range of neurologic sequelae, including weakness, headaches, and altered mental status. Given that patients with LM generally have quite poor prognoses, the decision of how to manage this HC remains unclear and is not only a medical, but also an ethical one.

Methods We first provide a brief overview of management options for hydrocephalus secondary to LM. We then apply general ethical principles to decision making in LM-associated hydrocephalus that can help guide physicians and patients. Results Management options for LM-associated hydrocephalus include shunt placement, repeated lumbar punctures, intraventricular reservoir placement, endoscopic third ventriculostomy, or pain management alone without intervention. While these options may offer symptomatic relief in the short-term, each is also associated with risks to the patient. Moreover, data on survival and quality-of-life following intervention is sparse. We propose that the pros and cons of each option should be evaluated not only from a clinical standpoint, but also within a larger framework that incorporates ethical principles and individual patient values.

Conclusions The decision of how to manage LM-associated hydrocephalus is complex and requires close collaboration amongst the physician, patient, and/or patient's family/friends/community leaders. Ultimately, the decision should be rooted in the patients' values and should aim to optimize a patient's quality-of-life.
\end{abstract}

Keywords Leptomeningeal metastases $\cdot$ Hydrocephalus $\cdot$ Ethics $\cdot$ Shunt $\cdot$ Quality-of-life

Marike L. Broekman

m.l.d.broekman@lumc.nl

1 Department of Neurosurgery, Computational Neurosciences Outcomes Center, Brigham and Women's Hospital, Boston, MA, USA

2 Harvard Medical School, Boston, MA, USA

3 Department of Neurosurgery, Brain Center Rudolf Magnus, University Medical Center Utrecht, Utrecht, The Netherlands

4 Department of Neurosurgery, Haaglanden Medical Center, The Hague, The Netherlands

5 Department of Neurosurgery, Leiden University Medical Center, PO Box 9600, 2300 RC Leiden, The Netherlands

6 Department of Neurology, Massachusetts General Hospital, Boston, MA, USA

\section{Introduction}

Leptomeningeal metastases (LM) are being increasingly encountered in present-day clinical practice. This greater incidence is likely due to advances in the treatment of cancer that allow patients to live long enough to develop late complications such as LM [1-3]. Yet, despite the progress in oncologic treatments, the prognosis for LM remains poor with average survival ranging from 3.5 to 6 months [1-4].

LM affects approximately 5-8\% of patients with solid tumors [5]. Patients with LM typically present with acute and often debilitating neurologic symptoms, including headaches, nausea, cognitive dysfunction, seizures, cranial nerve deficits, and/or weakness and pain $[2,4]$. As these symptoms greatly affect patients' quality-of-life [1], it is of importance to effectively manage them. Hydrocephalus (HC) secondary to LM is common [6], though outcomes in patients with metastases-related hydrocephalus are poor with approximately 10\% 1-year survival [4]. 
Management of hydrocephalus in patients with LM is challenging, as effective, immediate symptom relief often requires surgical intervention in frail cancer patients [4]. Although the available data exploring the effectiveness of ventriculoperitoneal shunt (VPS) placement in improving quality-of-life in this patient population is limited, the procedure is commonly performed to relieve patients of hydrocephalus-associated symptoms [1].

There is currently no clear medical consensus for the best treatment option to manage LM-associated hydrocephalus. We believe this question is less of a medical question and more of an ethical one. Given that the prognosis for patients with LM can vary based on performance status, systemic disease control, age, and primary tumor histology [3, 7], we believe that a more nuanced, patient-centered approach to managing their hydrocephalus should be undertaken. This approach should look not only at the hard facts surrounding expected disease course, but combine patient quality-of-life measures, cultural values, and fundamental ethical principles in determining the ideal management option for these patients.

Here, we aim to briefly present the current surgical options for LM-associated hydrocephalus and the pros and cons surrounding each. Subsequently, we will offer ways to facilitate physician and patient decision-making based on general ethical principles applied to patients with LMassociated hydrocephalus.

\section{Current surgical options}

Current options for management of LM-associated hydrocephalus include: (1) shunt placement; (2) repeated lumbar punctures; (3) intraventricular reservoir placement; (4) endoscopic third ventriculostomy; or (5) no intervention with focus on pain management $[3,4]$.

\section{CSF diversionary procedures: ventroperitoneal or lumbar shunting}

VPS involves placement of a catheter in the lateral ventricle, connected into the peritoneal cavity [8]. VPS allows for rapid normalization of elevated intracranial pressure (ICP) and is thus commonly employed to relieve symptoms of hydrocephalus $[5,8,9]$. Table 1 depicts studies that have previously reported symptoms and outcomes for patients with leptomeningeal disease-associated $\mathrm{HC}$ who have undergone shunting. Overall, these retrospective series have demonstrated significant improvement in hydrocephalus-associated symptoms following VPS placement, with rates of symptom improvement ranging from 75 to $>90 \%$ (Table 1) [4,
5, 9-11]. Shunting has also led to marked patient improvements in performance status, with post-surgical Karnofsky Performance Status (KPS) increases of $10-20$ points [4, 11]. While it has not been standard-of-care to intervene on patients with low performance scores, the minimal published data suggests that VPS may improve quality-of-life even in patients with low KPS $[9,10]$.

Despite the symptomatic relief following VPS experienced by many patients, there are gradations of benefit based upon various patient factors. For example, it appears that while headache and nausea are most likely to improve following VPS, results are variable with respect to cognitive dysfunction, urinary incontinence, and gait dysfunction $[9,11]$. Moreover, per the results of Murakami et al., only patients with a presenting KPS $\geq 30$ benefited from VPS. In contrast, those with a KPS $<30$ before surgery died within 1 month of their procedure due to worsening of their general condition [4]. In addition, while prior studies have shown that in general, patients with LMD from breast cancer, leukemia, and lymphoma tend to have slightly improved survival compared to LMD from other primary tumor sites [12,13], the studies in Table 1 that focused on patients with LMD-associated hydrocephalus did not find primary tumor histology to be prognostic for survival [5, 11]. However, the improvement in symptoms following VPS did allow for subsequent administration of systemic therapies in some cases, possibly contributing to increased survival $[1,11]$. These results suggest that in patients with primary tumors for which systemic therapies are available, alleviation of debilitating symptoms via placement of a shunt may allow for more rigorous treatment.

VPS also poses potential risks, including infection, bleeding, and peritoneal tumor dissemination, although none of the studies in Table 1 found any cases of peritoneal carcinomatosis after VPS $[1,5]$. These studies have demonstrated complication rates between 9 and 19\% [5, $9,10]$, often leading to a second surgery for shunt revision $[4,5,11]$.

As seen in Table 1, the data on outcomes following VPS in patients with LMD is sparse, and there is thus no obvious paradigm for clinicians to follow when recommending shunt surgery for LM-associated hydrocephalus. Even after VPS, median survival across studies remains low, ranging from 2 to 7.5 months (Table 1). Only one prior study had a "non-surgical" group to compare to the shunted patients, and while there was improved survival in the group that underwent shunting, this difference was non-significant, and there was also no data on symptom relief or quality-of-life to compare across the two groups. Thus, while those with severe presenting symptoms of headaches and nausea may derive the greatest benefit, more conservative approaches may be suitable for those with cognitive symptoms that are unlikely to improve or 


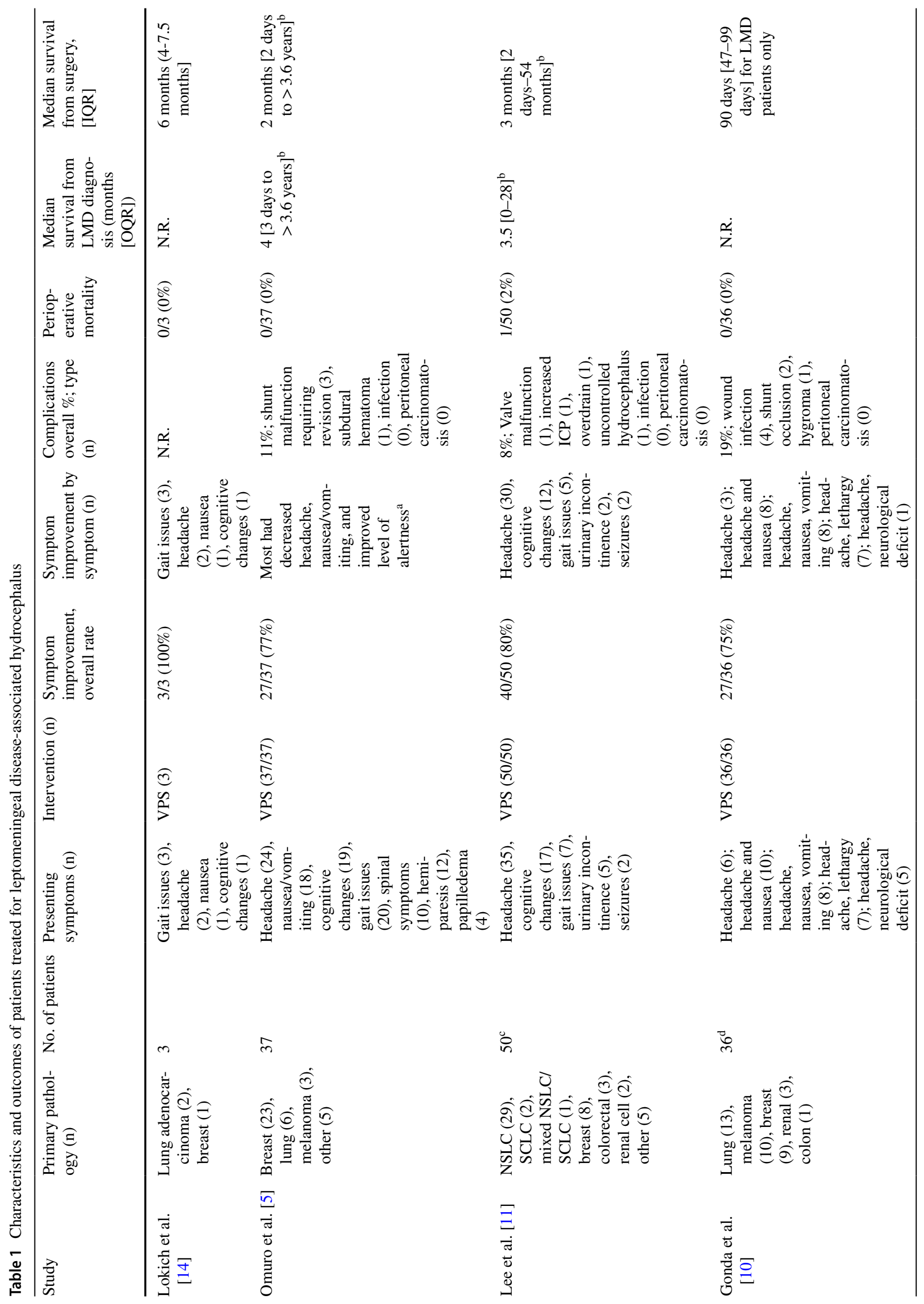




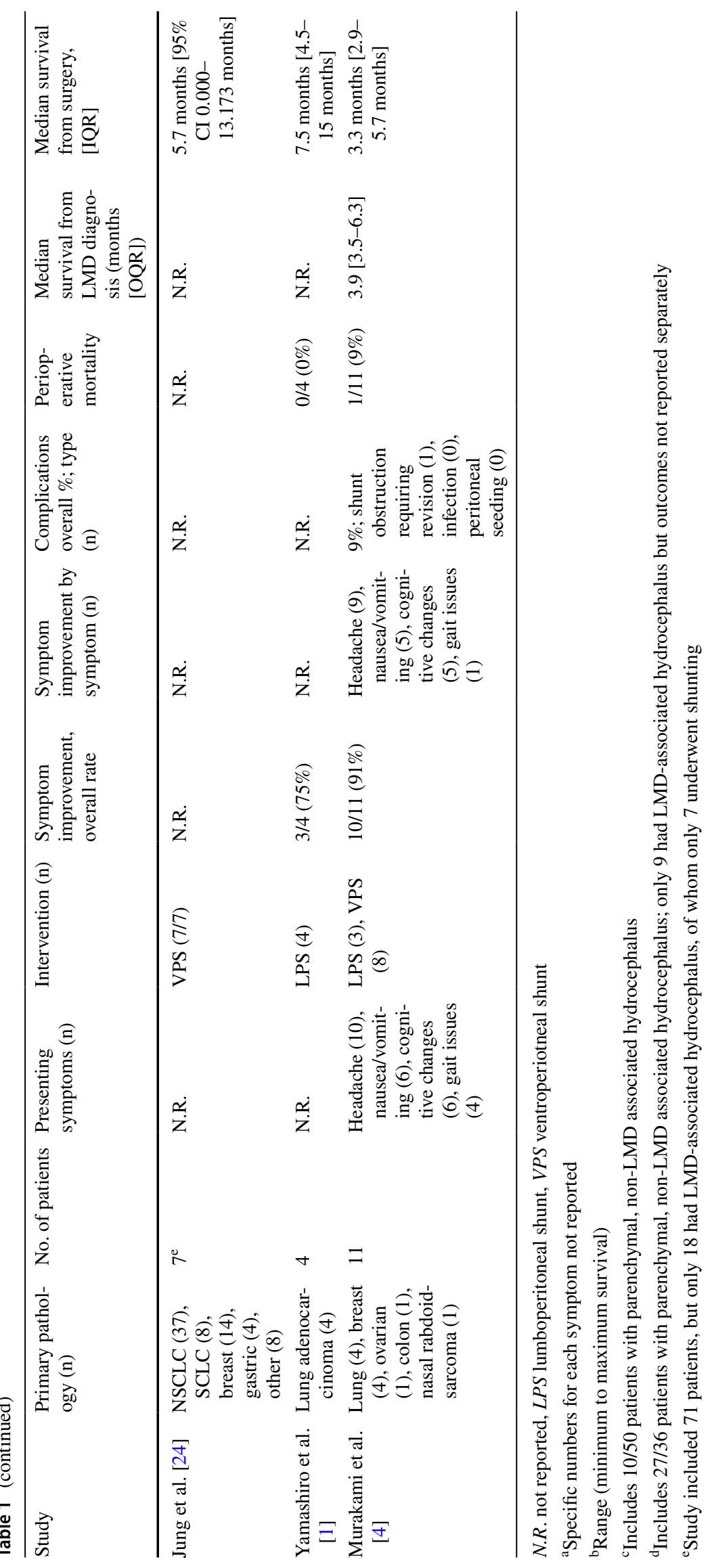


those with very low KPS. In addition, in the studies that did report on complications, the risks were non-negligible, and often led to revision surgery, which might pose significant challenges in a population of patients that is seriously ill and with poor reserve to begin with.

\section{Repeated lumbar punctures or placement of intraventricular reservoir}

Other potential options for relieving elevated ICP secondary to hydrocephalus involve repeated lumbar punctures (LPs) or the placement of an intraventricular reservoir [3, 15]. While typically performed to obtain CSF for diagnostic purposes, LPs have also been shown to result in rapid improvement of acute symptoms secondary to hydrocephalus $[8,16]$. However, serial LPs are not ideal as they subject patients to the constant discomfort of repeated intervention.

Intraventricular reservoirs, in contrast, work similarly to LPs, but are less imposing on patients. Such reservoirs are placed underneath the scalp in the frontal horn of the right lateral ventricle; a connecting catheter allows for communication between the device and CSF. Generally used for delivery of intrathecal chemotherapy in patients with leptomeningeal disease (LMD), they can also allow for CSF aspiration in the setting of high ICP $[15,17]$. Compared to LPs, repeated access to CSF is easier and more comfortable for patients with a reservoir.

However, reservoirs also come with possible complications, including infection, hemorrhage, or malpositioning $[8,17]$. Malpositioning rates range from 2.7 to $12.5 \%$ and can have serious consequences $[8,17,18]$. If a patient with hydrocephalus is not receiving chemotherapy, the utility for such an intervention compared to more permanent solutions must be weighed against the burden of an additional procedure that may eventually require conversion to a more sustainable solution anyways.

\section{Endoscopic third ventriculostomy}

LMD can present with either obstructive or non-obstructive hydrocephalus [5]; while VPS remains the preferred treatment in patients with non-obstructive hydrocephalus, endoscopic third ventriculostomy (ETV) may be another potential therapeutic option in those with obstructive hydrocephalus $[17,19]$. ETV involves the endoscopic placement of a small hole at the floor of the third ventricle that subsequently allows for diversion of CSF into the interpeduncular cistern $[17,20]$. It is minimally invasive, has a low operative morbidity, and is generally less uncomfortable for patients compared to VPS [19, 21]. LMD impairs CSF resorption capacity, and ETV would therefore not be expected to be efficacious in such cases of communicating HC [19]. The use of ETV in patients with LMD-associated hydrocephalus has therefore not been well-studied, although a few reports have looked at its role in patients with hydrocephalus secondary to parenchymal brain metastases $[10,19,21]$. These studies have demonstrated symptom improvement rates of $~ 70 \%$ in these patients, with lower or similar complication rates as compared to those following VPS [10, 19, 21].

One study triaged patients with hydrocephalus secondary to cerebral metastasis to either VPS or ETV based upon whether they had non-obstructive or obstructive hydrocephalus, respectively, and showed similar clinical outcomes in terms of symptom palliation, post-operative KPS improvement, and overall survival between the two groups [10]. While the difference in the overall complication rate between the two groups did not reach statistical significance, VPS patients had a greater complication rate of 19.4 versus $12.6 \%$ in the ETV group [10]. These results suggest that in patients with metastasis-related, obstructive hydrocephalus, the less invasive option of ETV might offer a similar degree of benefit to patients as VPS, but without as many complications. While the aforementioned studies did not assess a role for ETV in patients with LMD, their results suggest ETV may be an effective option when hydrocephalus is secondary to bulky LMD impairing CSF outflow [5]. Each patient's radiographic abnormalities should thus be studied to determine the cause of the hydrocephalus and subsequently to determine whether ETV might be a more suitable option than VPS in these patients. Overall, further study of ETV in patients with LMD is warranted.

\section{Non-intervention}

Patients may also opt not to treat their hydrocephalus. While the above studies demonstrate the effectiveness of surgical intervention on immediate symptom relief, the prognosis even after treatment of metastasis-related hydrocephalus remains poor [9]. Thus, the burden of an additional intervention may not be desirable. Median survival in patients with untreated LM ranges from 1 to 2 months, sometimes extending to 6 months in aggressively-treated populations [21-24]. While many of these patients die from progression of their systemic cancer [25], per some reports in the literature, the majority of deaths in this patient population occur due to progressive neurological involvement of the patients' disease [22, 23], underscoring the importance of determining whether neurosurgical intervention is justified if it is the neurological sequelae that are leading to patient death. A study by Jung et al. explored the prognostic significance of surgically treated hydrocephalus in LM and found improved overall survival in surgically treated hydrocephalus as compared to surgically untreated hydrocephalus (median 5.7 versus 1.7 months), although this did not reach statistical 
significance [24]. While this study did not evaluate qualityof-life or quality-of-death, studies should assess the comfort and pain levels of patients who live longer following surgical intervention for hydrocephalus. This information could allow clinicians and patients to better weigh the risks and benefits of intervention versus non-intervention.

\section{Ethics of intervention}

The lack of clear medical consensus on how to manage LMassociated hydrocephalus likely stems from a lack of robust data, as well as wide variation in patient presentation, belief systems, and environmental resources. Together, these make it difficult to recommend a general paradigm applicable to all patients. Patients' prognoses differ based upon prior and concurrent treatments, performance status, systemic disease burden, primary tumor histology, and other factors [3, 7]. Still, most patients with LM tend to be seriously ill and generally have poor prognoses. This complicates the decision to actively intervene at the risk of exposing the patient to further complications versus focusing on comfort measures alone.

As mentioned above, there is data to suggest prolonged survival following treatment of LM-associated hydrocephalus [24]. However, assessment of patient QOL during this extended survival period is lacking. Moreover, given that even after the surgery, median survival is still on the order of months, we think that fundamental ethical principles as opposed to statistics can better guide decision-making.

Beauchamp and Childress have previously proposed a four-principles approach to medical ethics, highlighting the following ethical principles: respect for autonomy; nonmaleficence; beneficence; and justice [26]. Below, we will briefly define these principles and apply them to LM-associated hydrocephalus.

\section{Respect for a patient's autonomy}

Respect for autonomy means that the physician accepts the choice made by a patient with decision-making capacity [26]. As patients with LM may have poor performance status and possibly neurocognitive sequelae [2], it is essential that the decision-making capacity of the patient is assessed early. Moreover, patients with LMD are generally terminally ill and comprise a particularly vulnerable group of individuals [27]. Some have previously suggested that decision-making capacity is impaired in patients who are nearing death, making true informed consent particularly challenging to obtain $[28,29]$. Furthermore, terminally ill patients often feel a sense of desperation stemming from unbearable symptoms and a hope to try any last effort to relieve suffering. Such desperation limits the voluntariness of any major decisionmaking $[28,30]$.
Thus, before the various options for management are even discussed with the patient, the physician should assess his or her competence. If the patient demonstrates full decisionmaking capacity, it is up to the team to clearly explain each of the above possible interventions, as well as their expected short-term and long-term consequences, so that the patient can make an informed decision. If a patient does not demonstrate decision-making capacity, a proxy can be used to make decisions on their behalf [29, 31]. When no proxy has been named, these decisions are typically left to family members, physicians, and hospitals, who are expected to practice "substituted judgment," i.e. to make a decision as the ill individual would have if they had been able to decide themselves [32].

Since each of the above options for LM-associated hydrocephalus will have different consequences for the patient's life, the patient's personal values should dictate the courseof-action. Therefore, it is essential to assess the capacity of the patient and ensure that either the patient, if capable, or someone familiar with the patient's belief systems be at the forefront of the decision-making process.

Studies have shown that patients' values and religion may have particularly strong influences on end-of-life planning. For example, Hindus and Buddhists typically prefer dying at home, which may ultimately dictate what types of treatments they choose to receive [33]. To illustrate, if the placement of a shunt allows a patient to return home because they no longer have uncontrollable nausea requiring a nasogastric tube, that patient may choose to undergo shunting even if it means they will have to undergo a potentially risky surgical intervention.

Physicians treating patients with LM face challenging decisions. They should first prioritize establishing patient capacity, and once established, they should show utmost respect for the patient's autonomy, rooted in his or her personal values, religious systems, and/or cultural paradigms. Through a shared decision-making approach that brings together medical information from the physician with the belief systems inherent to the patient, an optimal plan individual to that patient can be reached.

\section{Non-maleficence}

Non-maleficence means that physicians have the obligation to avoid harm to their patients [26, 34]. Omitting a standardof-care and exposing a patient to undue harm violates the principle of non-maleficence [34]. We believe that it is the responsibility of the physician and patient to work together to determine the course of action that minimizes harm to the patient while staying true to the patient's own values, as described above.

Depending on the patients' beliefs, he or she may favor relief in the short-term over long-term suffering, while 
others may view this as burdensome prolonging of life (or even suffering). Such views on what course of action is "less bad" are often influenced by patient religion and culture. For example, in Roman Catholicism, "Natural Law," is used to determine how to act morally in complex situations that may have both good and bad consequences [33]. Overall, an action with a negative consequence may be allowed if some greater good also occurs as a result of the action. For example, shortening of life may be permissible if it allows for alleviation of suffering. In the context of shunting in LM, such a belief system may prompt a patient to choose not to undergo intervention and opt for increasing pain medications instead, even if this means hastening death [33].

The theme once again emerges that in our case of leptomeningeal metastases, the decision of whether or not to intervene is unclear. However, what is clear is that a discussion with the patient and what he or she views as more or less "bad" should be undertaken to uphold the principle of non-maleficence and minimize harm to the patient, whether that be in the form of present-day or later suffering.

\section{Beneficence}

Per the principle of beneficence, physicians have an obligation to benefit their patients and to balance these benefits against any risks [26, 34]. Given the relative sparsity of data on quality-of-life measures following treatment for LM-associated hydrocephalus, upholding the principle of beneficence in such patients involves open discussion of all options, consideration of each patient's individual prognosis, as well as the patient's overall hopes and goals for any treatment. A patient with a KPS of 20 who is too weak to withstand another intervention, is unlikely to benefit from surgery. On the contrary, a young patient with a KPS of 70 who wishes to play an active role in their children's lives but is debilitated by headaches and nausea would likely benefit from intervention. Beneficence, or what is best for one patient, may be different from what is best for another; thus, physicians should follow an individualized course for each patient that optimizes his or her health and comfort while minimizing any associated harms.

\section{Justice}

The fourth principle of biomedical ethics is justice, which pertains to ensuring fairness in how health benefits are distributed in society [26]. While this principle has typically been explored in the context of situations in which there is a scarcity of resources (e.g. organ transplantation, rationing of intensive care unit beds [35]), we think it is applicable in any medical setting.
Numerous studies have demonstrated that physicians may exhibit implicit bias against certain patient populations based on race, sex, or other characteristics, and that this implicit bias has been linked to poorer communication, lower quality care, and differential treatment of these groups [35-38]. While not directly related to the allocation of tangible medical resources, such disparities and inequities in patient care violate the principle of justice.

Extrapolating this data to our case, it is important for physicians to maintain the same thoroughness in communication with all LM patients, regardless of race, sex, or education-level. In fact, attention to justice may be even more critical in cases involving LM, where the pathophysiology is particularly complicated and may be difficult for physicians to explain to laypersons. By leaving out information for some patients and thereby preventing those patients from having all the necessary data to make a decision, the physician would be violating the principle of justice. Thus, we believe that a key piece of the decision-making paradigm in how to manage LM-associated hydrocephalus requires the physician to spend ample time with and to equip each patient with a thorough enough understanding from which to make a decision.

\section{Shared decision making}

Ultimately, a decision as complex as how to manage LMassociated hydrocephalus requires open discussion and collaboration amongst the patient, patient's family/close friends, and physician. First and foremost, we believe that the physician needs to establish the decision-making capacity of the patient. Once clear who the decision-maker for the patient will be (either the patient or a proxy), the physician should communicate all options to this individual and any other individuals whom the patient wishes. It is the responsibility of the physician to be complete and unbiased in presenting the objective risks and benefits of each option, tailored to that patient's individual prognosis and disease presentation. Once this "factual" baseline is established, the physician should learn the patient's values, cultural beliefs, and personal priorities. In situations where it may be helpful for the patient, religious or community leaders may also be involved to weigh in on the decision.

Recently, there has also been an increasing amount of evidence that supports the early involvement of palliative care teams in the care of cancer patients [39]. In those with terminal illnesses, these teams are specially trained to focus on caring for patients in a way that aligns with their values. Moreover, palliative care specialists, because of their interdisciplinary nature, are optimally positioned to facilitate communication among the many teams taking care of cancer patients [39]. Indeed, data from multiple randomized controlled trials have demonstrated that when palliative care 
is integrated with routine oncological care, patients report improved quality-of-life, less aggressive end-of-life care, reduced symptom severity, and improved prognostic awareness [38-42]. Based on these promising reports, palliative care physicians should be increasingly integrated into this shared decision-making model, as well.

With both the objective data set forth by the physician and the belief system of the patient made apparent, the two should work with teams like the palliative care doctors to re-assess the options and finally come to a shared decision. This shared decision should aim to respect the patient's autonomous choices, while also staying true to the ethical medical principles of doing good and minimizing harm. By listening to and engaging with their patients, physicians can prioritize the patient's dignity and comfort at a time of deep suffering and pain.

\section{Conclusions}

LM-associated hydrocephalus severely impacts length and quality of life. Given the paucity of quality-of-life and survival data, there is currently no consensus in the medical community regarding how best to manage hydrocephalus in these patients. Treatment options include (1) shunt placement; (2) repeat lumbar punctures; (3) intraventricular reservoir placement; (4) endoscopic third ventriculostomy; or (5) no acute intervention with a focus on pain management. We believe that the four ethical principles put forth by Beauchamp and Childress (autonomy, non-maleficence, beneficence, and justice) can aid in analysis of how to manage LM-associated hydrocephalus. Furthermore, we believe that the management of LM-hydrocephalus should hinge upon rigorous upholding of these principles as well a highly individualized, patient-centered discussion of the management options. In this way, patients with a serious illness can safely and strongly live out whatever time they have left in a matter consistent with their personal and familial values.

\section{Compliance with ethical standards}

Conflict of interest The authors declare that they have no conflict of interest.

Informed consent This research did not involve humans, and no informed consent was required.

Research involving human participants and/or animals This research did not involve human participants or animals.

Open Access This article is distributed under the terms of the Creative Commons Attribution 4.0 International License (http://creativeco mmons.org/licenses/by/4.0/), which permits unrestricted use, distribution, and reproduction in any medium, provided you give appropriate credit to the original author(s) and the source, provide a link to the Creative Commons license, and indicate if changes were made.

\section{References}

1. Yamashiro S, Hitoshi Y, Tajiri S, Uchikawa H, Ito K, Yoshida A, Kuratsu JI (2017) Palliative lumboperitoneal shunt for leptomeningeal metastasis-related hydrocephalus: a case series. Palliat Med 31(1):93-96. https://doi.org/10.1177/0269216316649128

2. Leal T, Chang JE, Mehta M, Robins HI (2011) Leptomeningeal metastasis: challenges in diagnosis and treatment. Curr Cancer Ther Rev 7(4):319-327. https://doi.org/10.2174/1573394117 97642597

3. Jaeckle KA (2006) Neoplastic meningitis from systemic malignancies: diagnosis, prognosis and treatment. Semin Oncol 33(3):312323. https://doi.org/10.1053/j.seminoncol.2006.04.016

4. Murakami Y, Ichikawa M, Bakhit M, Jinguji S, Sato T, Fujii M, Sakuma J, Saito K (2018) Palliative shunt surgery for patients with leptomeningeal metastasis. Clin Neurol Neurosurg 168:175-178. https://doi.org/10.1016/j.clineuro.2018.03.008

5. Omuro AM, Lallana EC, Bilsky MH, DeAngelis LM (2005) Ventriculoperitoneal shunt in patients with leptomeningeal metastasis. Neurology 64(9):1625-1627. https://doi. org/10.1212/01.WNL.0000160396.69050.DC

6. Grossman SA, Krabak MJ (1999) Leptomeningeal carcinomatosis. Cancer Treat Rev 25(2):103-119. https://doi.org/10.1053/ ctrv.1999.0119

7. Roth P, Weller M (2015) Management of neoplastic meningitis. Chin Clin Oncol 4(2):26. https://doi.org/10.3978/j. issn.2304-3865.2015.05.02

8. Drappatz J, Batchelor TT (2007) Leptomeningeal neoplasms. Curr Treat Options Neurol 9(4):283-293

9. Nigim F, Critchlow JF, Kasper EM (2015) Role of ventriculoperitoneal shunting in patients with neoplasms of the central nervous system: an analysis of 59 cases. Mol Clin Oncol 3(6):1381-1386. https://doi.org/10.3892/mco.2015.627

10. Gonda DD, Kim TE, Warnke PC, Kasper EM, Carter BS, Chen CC (2012) Ventriculoperitoneal shunting versus endoscopic third ventriculostomy in the treatment of patients with hydrocephalus related to metastasis. Surg Neurol Int 3:97. https://doi. org/10.4103/2152-7806.100185

11. Lee SH, Kong DS, Seol HJ, Nam DH, Lee JI (2011) Ventriculoperitoneal shunt for hydrocephalus caused by central nervous system metastasis. J Neurooncol 104(2):545-551. https://doi. org/10.1007/s11060-010-0512-2

12. Hildebrand $\mathbf{J}$ (1998) Prophylaxis and treatment of leptomeningeal carcinomatosis in solid tumors of adulthood. J Neurooncol 38(2-3):193-198

13. DeAngelis LM (1998) Current diagnosis and treatment of leptomeningeal metastasis. J Neurooncol 38(2-3):245-252

14. Lokich J, Levine H, Nasser I (1998) Malignancy-related hydrocephalus: clinical features and results ofventricular peritoneal shunt procedure in three patients. Am J Clin Oncol 21(4):366-368

15. Pople IK (2002) Hydrocephalus and shunts: what the neurologist should know. J Neurol Neurosurg Psychiatry 73(Suppl 1):i17-i22

16. Hasan D, Lindsay KW, Vermeulen M (1991) Treatment of acute hydrocephalus after subarachnoid hemorrhage with serial lumbar puncture. Stroke 22(2):190-194

17. Volkov AA, Filis AK, Vrionis FD (2017) Surgical Treatment for Leptomeningeal Disease. Cancer Control 24(1):47-53. https://doi. org/10.1177/107327481702400107 
18. Sandberg DI, Bilsky MH, Souweidane MM, Bzdil J, Gutin PH (2000) Ommaya reservoirs for the treatment of leptomeningeal metastases. Neurosurgery 47(1):49-54 (discussion 54-45)

19. Chen CC, Kasper E, Warnke P (2011) Palliative stereotacticendoscopic third ventriculostomy for the treatment of obstructive hydrocephalus from cerebral metastasis. Surg Neurol Int 2:76. https://doi.org/10.4103/2152-7806.82083

20. Yadav YR, Parihar V, Pande S, Namdev H, Agarwal M (2012) Endoscopic third ventriculostomy. J Neurosci Rural Pract 3(2):163-173. https://doi.org/10.4103/0976-3147.98222

21. Nguyen TT, Smith MV, Rodziewicz GS, Lemke SM (1999) Hydrocephalus caused by metastatic brain lesions: treatment by third ventriculostomy. J Neurol Neurosurg Psychiatry 67(4):552-553

22. Balm M, Hammack J (1996) Leptomeningeal carcinomatosis. Presenting features and prognostic factors. Arch Neurol 53(7):626-632

23. Grossman SA, Finkelstein DM, Ruckdeschel JC, Trump DL, Moynihan T, Ettinger DS (1993) Randomized prospective comparison of intraventricular methotrexate and thiotepa in patients with previously untreated neoplastic meningitis. Eastern cooperative oncology group. J Clin Oncol 11(3):561-569. https://doi. org/10.1200/JCO.1993.11.3.561

24. Jung TY, Chung WK, Oh IJ (2014) The prognostic significance of surgically treated hydrocephalus in leptomeningeal metastases. Clin Neurol Neurosurg 119:80-83. https://doi.org/10.1016/j.cline uro.2014.01.023

25. Berg SL, Chamberlain MC (2003) Systemic chemotherapy, intrathecal chemotherapy, and symptom management in the treatment of leptomeningeal metastasis. Curr Oncol Rep 5(1):29-40

26. Beauchamp TL (2003) Methods and principles in biomedical ethics. J Med Ethics 29(5):269-274

27. Shivayogi P (2013) Vulnerable population and methods for their safeguard. Perspect Clin Res 4(1):53-57. https://doi. org/10.4103/2229-3485.106389

28. Casarett DJ, Karlawish JH (2000) Are special ethical guidelines needed for palliative care research? J Pain Symptom Manage 20(2):130-139

29. Agar M, Ko DN, Sheehan C, Chapman M, Currow DC (2013) Informed consent in palliative care clinical trials: challenging but possible. J Palliat Med 16(5):485-491. https://doi.org/10.1089/ jpm.2012.0422

30. Cote DJ, Balak N, Brennum J, Holsgrove DT, Kitchen N, Kolenda H, Moojen WA, Schaller K, Robe PA, Mathiesen T, Broekman ML (2017) Ethical difficulties in the innovative surgical treatment of patients with recurrent glioblastoma multiforme. J Neurosurg 126(6):2045-2050. https://doi.org/10.3171/2016.11.JNS162488

31. Cohen NH, Kummer HB (2006) Ethics update: lessons learned from Terri Schiavo: the importance of healthcare proxies in clinical decision-making. Curr Opin Anaesthesiol 19(2):122-126. https://doi.org/10.1097/01.aco.0000192774.24774.5c

32. McDade-Montez E, Watson D, Beer A (2013) Similarity, agreement, and assumed similarity in proxy end-of-life decision making. Fam Syst Health 31(4):366-381. https://doi.org/10.1037/ a0033372

33. Setta SM, Shemie SD (2015) An explanation and analysis of how world religions formulate their ethical decisions on withdrawing treatment and determining death. Philos Ethics Humanit Med 10:6. https://doi.org/10.1186/s13010-015-0025-x

34. McCormick TR (2013) Principles of bioethics. University of Washington. https://depts.washington.edu/bioethx/tools/princ pl.html

35. Scheunemann LP, White DB (2011) The ethics and reality of rationing in medicine. Chest 140(6):1625-1632. https://doi. org/10.1378/chest.11-0622

36. Todd KH, Samaroo N, Hoffman JR (1993) Ethnicity as a risk factor for inadequate emergency department analgesia. JAMA 269(12):1537-1539

37. Heins JK, Heins A, Grammas M, Costello M, Huang K, Mishra S (2006) Disparities in analgesia and opioid prescribing practices for patients with musculoskeletal pain in the emergency department. J Emerg Nurs 32(3):219-224. https://doi.org/10.1016/j. jen.2006.01.010

38. Chapman EN, Kaatz A, Carnes M (2013) Physicians and implicit bias: how doctors may unwittingly perpetuate health care disparities. J Gen Intern Med 28(11):1504-1510. https://doi.org/10.1007/ s11606-013-2441-1

39. Hui D, Bruera E (2016) Integrating palliative care into the trajectory of cancer care. Nat Rev Clin Oncol 13(3):159-171. https:// doi.org/10.1038/nrclinonc.2015.201

40. Zimmermann C, Swami N, Krzyzanowska M, Hannon B, Leighl N, Oza A, Moore M, Rydall A, Rodin G, Tannock I, Donner A, Lo C (2014) Early palliative care for patients with advanced cancer: a cluster-randomised controlled trial. Lancet 383(9930):1721-1730. https://doi.org/10.1016/S0140-6736(13)62416-2

41. Temel JS, Greer JA, Muzikansky A, Gallagher ER, Admane S, Jackson VA, Dahlin CM, Blinderman CD, Jacobsen J, Pirl WF, Billings JA, Lynch TJ (2010) Early palliative care for patients with metastatic non-small-cell lung cancer. N Engl J Med 363(8):733742. https://doi.org/10.1056/NEJMoa1000678

42. Temel JS, Greer JA, Admane S, Gallagher ER, Jackson VA, Lynch TJ, Lennes IT, Dahlin CM, Pirl WF (2011) Longitudinal perceptions of prognosis and goals of therapy in patients with metastatic non-small-cell lung cancer: results of a randomized study of early palliative care. J Clin Oncol 29(17):2319-2326. https:// doi.org/10.1200/JCO.2010.32.4459 